\title{
Research and development of dye-sensitized solar cells in the Center for Molecular Devices: from molecules to modules
}

\author{
Gerrit Boschloo ${ }^{1, *}$, Anders Hagfeldt ${ }^{1}$, Håkan Rensmo ${ }^{1}$, Lars Kloo ${ }^{2}$, Licheng Sun ${ }^{2}$, Henrik \\ Pettersson ${ }^{3}$ \\ ${ }^{1}$ Uppsala University, Uppsala, Sweden \\ ${ }^{2}$ Royal Institute of Technology, Stockholm, Sweden \\ ${ }^{3}$ Swerea IVF, Mölndal, Sweden \\ *Corresponding author.Tel: +46184713303,E-mail: gerrit.boschloo@fki.uu.se
}

\begin{abstract}
Dye-sensitized solar cells (DSCs) represent a relatively new photovoltaic technology with great potential: investment costs for initiating production are low and manufacturing costs below $0.5 \mathrm{US} \$ / \mathrm{W}_{\text {peak }}$ are predicted. Furthermore, DSC offers the possibility of various colors and attractive designs, such as semitransparent modules. Record solar cell efficiencies are $11 \%$ for DSCs containing liquid redox electrolyte and $6 \%$ for DSCs with solid hole conductors. Promising stability data suggesting more than 20 years lifetime has been achieved. This paper presents the Center for Molecular Devices (CMD) in Sweden, which has as its objective to investigate and develop DSCs. Using a multi-disciplinary approach, significant advances in the scientific understanding of DSCs have been made, such as the demonstration of the presence of an internal electric field at the semiconductor / dye / electrolyte interface. Furthermore, novel components, such as triphenylamine-based dyes and cobalt-based mediator have been successfully tested. Finally, a monolithic DSC module technology with good performance is presented.
\end{abstract}

Keywords: Dye-sensitized solar cell, PV modules.

\section{Introduction}

Dye-sensitized solar cells (DSCs) represent a relatively new photovoltaic technology with great potential.[1] Certified record solar cell efficiencies are $11 \%$ for DSCs containing liquid redox electrolyte and $6 \%$ for DSCs with solid hole conductors. It is believed that this technology has the potential to reach production costs as low as $0.5 \mathrm{US} \$ / \mathrm{W}_{\text {peak, }}$, [2] which would make solar electricity generation competitive with conventional (fossil fuel based) electricity generation. Module efficiencies of $10 \%$ and lifetimes of 15 year were assumed for this calculation. More recent calculations, based on actual DSC devices and current material costs, give a manufacturing cost of $2.5 \mathrm{Euro} / \mathrm{W}_{\text {peak }}$, which may decrease to less than 1 Euro/ $\mathrm{W}_{\text {peak }}$ taking the expected decrease in material cost into account.[3]

Dye-sensitized solar cells differ much from conventional solid state semiconductor based solar cells.[1] Dye molecules, rather than an inorganic semiconductor material, are responsible for light absorption. Furthermore, the functions of light absorption, electron transport and hole transport are separated into different materials. Electron transport takes place in a porous $\mathrm{TiO}_{2}$ structure, while hole transport occurs in a liquid redox electrolyte. In its standard form the DSC consists of the following components (see Figure 1a):

- The working electrode: a mesoporous film of $\mathrm{TiO}_{2}$ nanoparticles ( $\mathrm{size} \sim 20 \mathrm{~nm}$ ) with a thickness of about $10 \mu \mathrm{m}$, on a fluorine-doped tinoxide (FTO) coated glass substrate. Dye-molecules are adsorbed at the surface of $\mathrm{TiO}_{2}$. The $\mathrm{TiO}_{2}$ framework acts as electron acceptor and transport medium. 
- A redox electrolyte: solution containing a suitable redox couple in a high concentration, as well as some additives that improve solar cell performance. The most common redox couple used in DSC is iodide / triiodide.

- A counter electrode: an electrode with good catalytic activity for electron transfer to the redox electrolyte. The most common counter electrode in DSCs is platinum-coated FTO-glass. This electrode has the advantage that it can be transparent, as a very low loading of Pt is needed.

- Sealing. Hermetic sealing is very important in order to obtain stable solar cells with good long term stability. The most common DSC sealing material is thermoplastic. A thermoplastic frame is used to connect the WE and CE together, which ensures a fixed separation between the two electrodes.

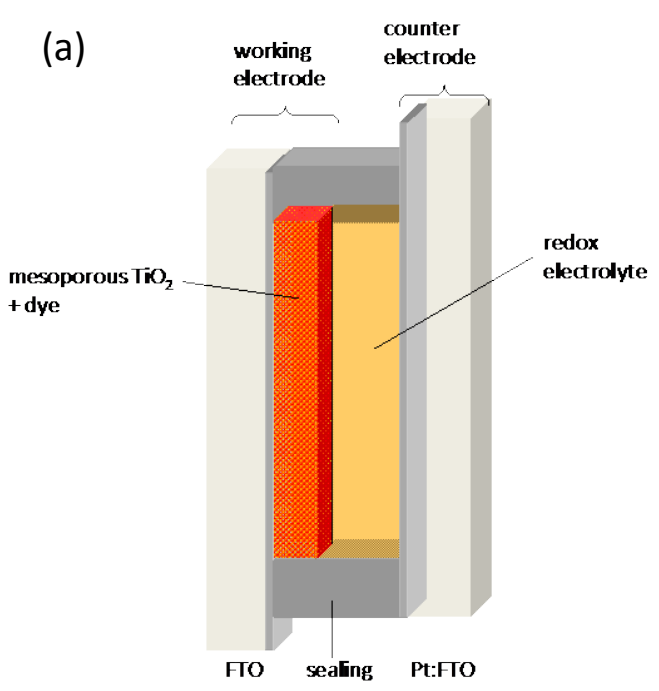

(b)

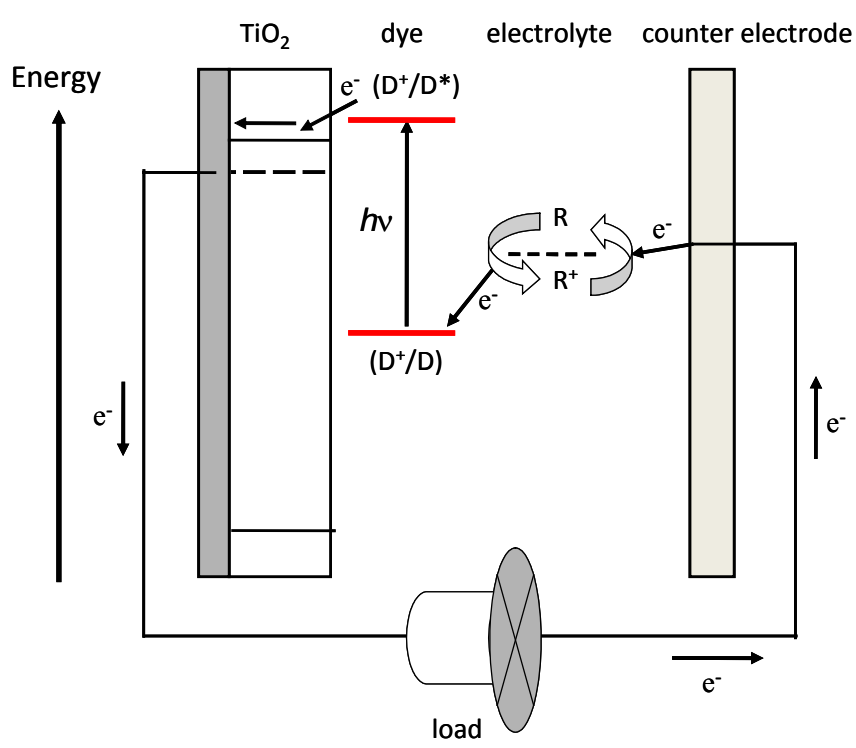

Figure 1. (a) Schematic structure of a dye-sensitized solar cell (sandwich design). FTO stands for fluorine-doped tinoxide-coated glass. (b) Energy scheme of a dye-sensitized solar cell. The arrows indicate electron transfer reactions.

The working mechanism of the dye-sensitized solar cell is displayed in Figure 1b. Light is absorbed by the dye molecules $\mathrm{D}$ resulting in excited dye molecules (D*). Ultrafast electron injection takes place from the excited dye into the conduction band of $\mathrm{TiO}_{2}$. Regeneration of the oxidized dye by the redox mediator R. Electrons are collected at the conducting substrates and can perform electrical work in an external circuit. At the counter electrode, electrons reduce $\mathrm{R}^{+}$, the oxidized form of mediator.

Despite intense research in the field of dye-sensitized solar cells, many fundamental aspects are still unclear. The Center for Molecular Devices (CMD) in Sweden has as its objective to investigate and develop DSCs. Using a multi-disciplinary approach, significant advances in the scientific understanding of DSCs, development of low-cost DSC components, and DSC module manufacturing have been made, as will be discussed here in this paper. 


\section{Methodology}

We refer to our research papers (ref. 4-17) for details on m esoporous $\mathrm{TiO}_{2}$ electrode preparation, dye synthesis, electrolyte preparation, solar cell assembly and solar cell characterization.

\section{Results and Discussion}

\subsection{Design, synthesis and characterization of dye molecules for DSC}

Research on dyes within CMD has been mainly focused on organic dyes with the general structure: donor - conjugated bridge - acceptor (D- $\pi$-A). Upon excitation, electron density will be displaced from the electron rich donor moiety towards the electron withdrawing acceptor moiety. The acceptor moiety is also equipped with suitable binding groups for attachment of the molecules onto the $\mathrm{TiO}_{2}$ surface. The general structure of $\mathrm{D}-\pi-\mathrm{A}$ dyes favors electron injection into $\mathrm{TiO}_{2}$ upon excitation, while the remaining positive charge will be located on the donor part, positioned relatively far away from the $\mathrm{TiO}_{2}$ surface. This will decrease the rate for direct geminate recombination. A very suitable donor group for $\mathrm{D}-\pi-\mathrm{A}$ dyes is the triphenylamine (TPA) unit, while a suitable acceptor / binding unit is cyanoacrylic acid.

In 2005 the D5 dye was developed within CMD as the first in a series of D- $\pi$-A dyes (see Figure 2a).[4] Although the absorption spectrum has a maximum at about $480 \mathrm{~nm}$ and does not extend significantly beyond $600 \mathrm{~nm}$, promising solar cell efficiencies of $6 \%$ were obtained in combination with the iodide / triiodide electrolyte. Other interesting results obtained with D5 are its suitability in solid-state DSCs, and its capacity for hole conduction when it is adsorbed on $\mathrm{TiO}_{2}$ as a monolayer.[5]

Several modifications of the D5 base structure have been synthesized and tested. One of the most promising dyes developed within CMD to date is the D35 dye (see Figure 2). The most prominent alteration is the addition of two $o$,p-dibutoxyphenyl groups on the TPA unit. This provides the dye with suitable steric properties, making the dye very suited to be used in combination with cobalt-based redox electrolytes [6] or in solid-state DSC.

(a)

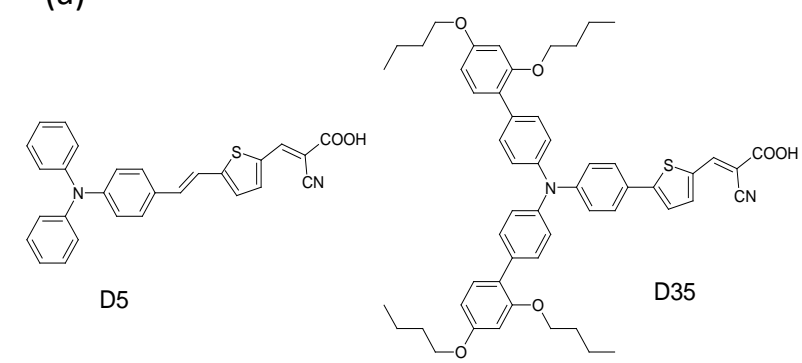

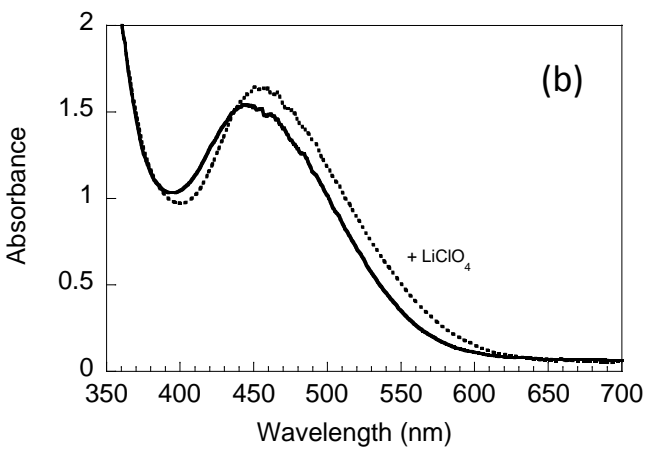

Figure 2. (a) Chemical structure of two efficient organic sensitizing dyes developed at CMD: D5 and D35. (b) UV-visible spectra of a D35-sensitized $\mathrm{TiO}_{2}$ film in air (drawn line) and in contact with $\mathrm{LiClO}_{4}$ in ethanol (dotted line). 


\subsection{Redox electrolytes and hole conductors}

The standard redox electrolyte for DSC comprises of the iodide / triiodide $\left(\mathrm{I}^{-} / \mathrm{I}_{3}{ }^{-}\right)$redox couple in an organic solvent. It is used in best performing DSCs to date, both in terms of efficiency and stability. It has good solubility, absorbs not too much visible light, it has a suitable redox potential, and provides rapid dye regeneration. But what $\mathrm{I}^{-} / \mathrm{I}_{3}{ }^{-}$makes particularly successful is the very slow recombination kinetics between electrons in $\mathrm{TiO}_{2}$ and the oxidized part of the redox couple, triiodide. A serious disadvantage of this redox mediator is that a significant part of the potential is lost due to intermediate reactions.[7] After electron injection, the oxidized dye $\left(D^{+}\right)$is reduced by iodide in the following way:

$\mathrm{D}^{+}+\mathrm{I}^{-} \rightarrow(\mathrm{D}-\mathrm{-})$

$(\mathrm{D}-\mathrm{-I})+\mathrm{I}^{-} \rightarrow \mathrm{D}+\mathrm{I}_{2}^{-\bullet}$

$2 \mathrm{I}_{2}^{-\bullet} \rightarrow \mathrm{I}_{3}^{-}+\mathrm{I}^{-}$

The diiodide radical $\left(\mathrm{I}_{2}^{-{ }^{-}}\right)$is produced as an intermediate in this process (reaction 2). We estimate that the disproportionation reaction 3 corresponds to an internal loss of potential of as much as $400 \mathrm{mV}$. We believe that it is this internal loss that is largely responsible for the observed 'stagnation' in record efficiencies obtained for DSCs.

In order to obtained efficiencies larger than $12 \%$, it may be necessary to using one-electron redox couples or hole conductors instead of $\mathrm{I}^{-} / \mathrm{I}_{3}{ }^{-}$, so that the losses due to internal conversion are avoided. Unfortunately, the use of one-electron mediators in DSC nearly always leads to strongly increased recombination between electrons in $\mathrm{TiO}_{2}$ and the oxidized part of the redox couple, which seriously limits the solar cell efficiency. Recently, however, we obtained a breakthrough with cobalt polypyridine-based mediators in combination with D35 dye.[6] Careful matching of the steric bulk of the mediator and the dye molecules minimizes the recombination between electrons in $\mathrm{TiO}_{2}$ and $\mathrm{Co}$ (III) species in the electrolyte and avoids mass transport limitations of the redox mediator. The organic sensitizer D35, equipped with bulky alkoxy groups, efficiently suppresses recombination, allowing the use of cobalt redox mediators with relatively small steric bulk. Its high extinction coefficient allows for making DSCs with thin $\mathrm{TiO}_{2}$ films, which is favorable with respect to charge recombination and mass transport of redox mediator in the porous structure. The best efficiency obtained for a DSC sensitized with D35 and employing a $\left[\mathrm{Co}(\mathrm{bpy})_{3}\right]^{3+/ 2+}$-based electrolyte was $6.7 \%$ at full sunlight (1000 $\mathrm{W} \mathrm{m}^{-2}$ AM1.5G illumination), which is more than a doubling of previously published record efficiencies using similar Co-based mediators. Notably, similar efficiencies with the D35 dye are obtained with $\mathrm{I}^{-} / \mathrm{I}_{3}{ }^{-}$as a redox couple.

The use of a solid-state hole conductors in DSCs is very attractive, but also very challenging. Besides the above mentioned problem of enhance recombination, an additional problem is to fill the pores of the dye-modified mesoporous $\mathrm{TiO}_{2}$ electrodes completely with the solid hole conductor. A possible solution investigated within CMD is melting infiltration of the hole conductor into the pores of the dye-sensitized $\mathrm{TiO}_{2}$ electrode.[8] The rather high temperatures required for melting is damaging for the organic dye molecules and so far is limiting the efficiencies of the resulting solar cells.

The most well-tested hole conductor in solid-state DSCs is 2,2',7,7'-tetrakis $(N, N$-di- $p$ methoxyphenylamine)-9,9'-spirobifluorene (spiro-MeOTAD). With the help of photoinduced absorption spectroscopy it was be shown that in principle all dye molecules appeared to be in contact with the hole conductor for a $6 \mu \mathrm{m}$ thick $\mathrm{TiO}_{2}$ film, even though pores are not filled to 
100\%.[9] A compelling result obtained within CMD research is that some perylene-based dyes performed much better in solid-state DSCs using spiro-MeOTAD than in standard iodide / triiodide electrolyte-based DSCs.[10] This can be attributed to the much faster regeneration kinetics observed in solid-state DSCs.

\subsection{Advanced characterization of DSC components and complete devices}

The dye-sensitized solar cell is a complex system with many interactions between its individual components. We have performed detailed characterization of dye-sensitized $\mathrm{TiO}_{2}$ films using advanced techniques such as X-ray photoelectron spectroscopy [11] and scanning tunneling microscopy,[12] giving valuable information of the binding morphology of the dyes and energy levels.

Investigations on complete DSC devices have been particularly fruitful, as the interactions between different components can be studied. For instance, we have studied the effect of the DSC electrolyte additive 4-tert butylpyridine in detail and found that it was responsible for a band edge shift of the $\mathrm{TiO}_{2}$ as well as for a reduction in the electron recombination rate constant.[13] More recently, we found that the additives guanidinium thiocyanate and $\mathrm{N}$ methylbenzimidazole have a s ynergistic effect on the solar cell performance of DSCs with ionic liquid $\left(\mathrm{I}^{-} / \mathrm{I}_{3}{ }^{-}\right)$electrolytes.[14]

Recently, we discovered that the Stark effect plays an important role in the transient absorption spectroscopy of DSCs.[15] The occurrence of the Stark effects implies that the electric field across the adsorbed dye molecules changes. The effect of electric fields can be even observed under steady-state conditions. Figure $2 \mathrm{~b}$ shows the absorption spectrum of D35 adsorbed on mesoporous $\mathrm{TiO}_{2}$. In the presence of lithium ions, a significant red shift of the spectrum is observed. This shift can be attributed to the $\mathrm{Li}^{+}$ions that adsorb onto the $\mathrm{TiO}_{2}$ surface, thus changing its surface charge. This in turn affects the electric field across the dye monolayer, giving rise to a Stark shift. The occurrence of this shift implies that the dyes are, at least partially, located within the Helmholtz double layer at the metal oxides / electrolyte interface. The effect of the electric field is that the donor-acceptor character of the dye is enhanced, resulting in a red-shift of the absorption spectrum.

\subsection{Monolithic DSC Modules}

Screen printing as is low cost, scalable method to prepare thin films. Within CMD, a monolithic design is used in the development of DSC modules, see Figure 3a.[16, 17] The monolithic design has several advantages: it can give significant cost reduction as only one FTO-coated glass plate is needed, compared to two in other (sandwich) designs. FTO substrates are responsible for up to $25 \%$ of the total manufacturing costs in a sandwich design DSC. Furthermore, problems with alignment and glass bending encountered for large sandwich-design modules are avoided.

In Figure $3 \mathrm{~b}$ s ome typical photocurrent density - voltage $(J-V)$ curves of a $13.5 \mathrm{~cm}^{2}$ sized DSC module are shown. The module consists of $4 \mathrm{c}$ ells connected in parallel. The solar to electrical power conversion efficiency depends on incident light intensity and ranges from $4.0 \%\left(1000 \mathrm{~W} \mathrm{~m}^{-2}\right)$ to $6.6 \%\left(56 \mathrm{~W} \mathrm{~m}^{-2}\right)$. This dependency is caused by internal resistance losses, of which the origin is currently under investigation. Stability tests as well as outdoor tests are ongoing. When the components in the DSC modules are well chosen (dye, electrolyte), the modules are completely stable after $2000 \mathrm{~h}$ in an accelerated aging test (1000 $\mathrm{W} \mathrm{\textrm {m } ^ { - 2 }}$ simulated sunlight, $50^{\circ} \mathrm{C}$ ), with an efficiency of $5.0 \%$ at $200 \mathrm{~W} \mathrm{~m}^{-2}$ illumination.[17] Significant degradation is, however, observed under storage in the dark at $80^{\circ} \mathrm{C}$. These tests 
were performed with the ruthenium-based complex K77 as the sensitizer and an iodide/ triiodide electrolyte with 3-methoxyproprionitrile as solvent.

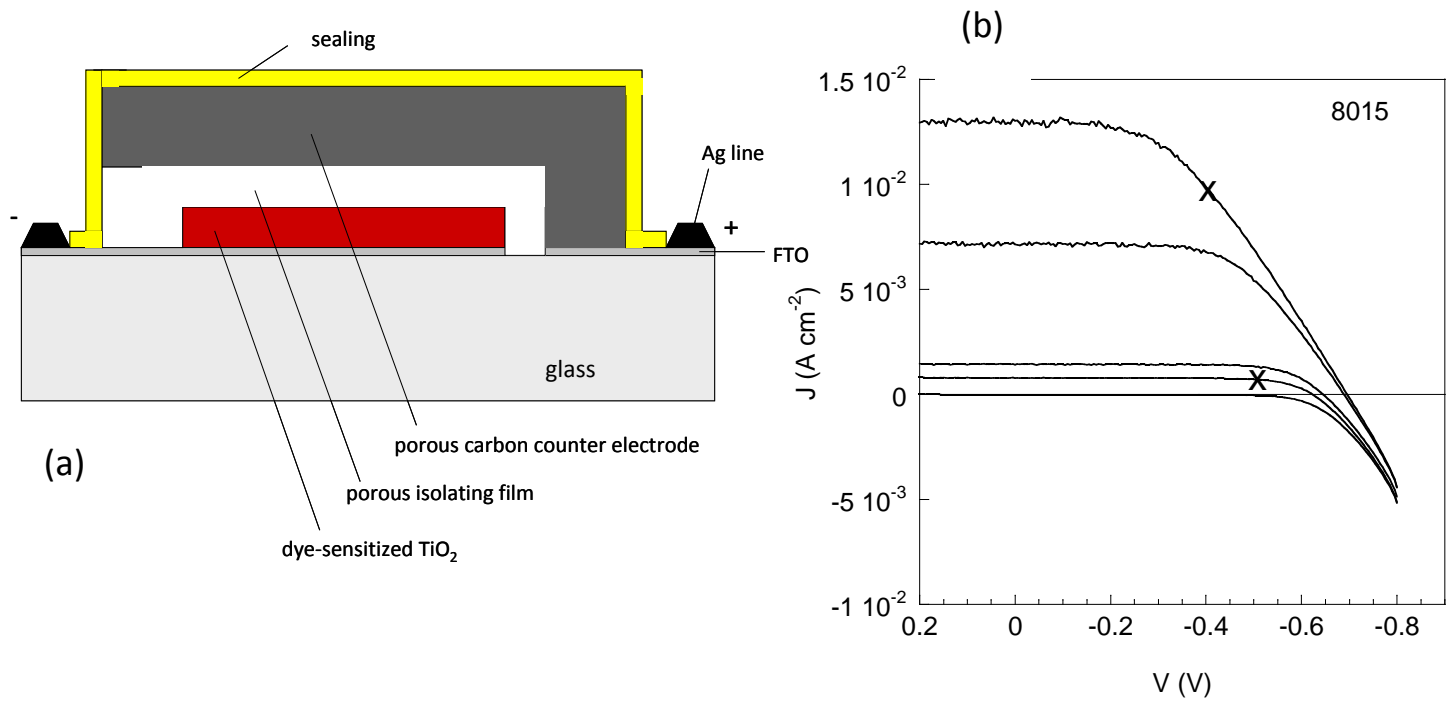

Figure 3. (a) Schematic structure of the monolithic design of the dye-sensitized solar cell.(b) J-V characteristics of a monolithic DSC module (active area: $13.5 \mathrm{~cm}^{2}$ ).Light intensities: 1000; 534; 105; 56 and $0 \mathrm{Wm}^{-2}$.

\section{Conclusions}

The work performed at the Center for Molecular Devices in Sweden has significantly contributed to the research field of dye-sensitized solar cells. CMD was among the first to develop organic sensitizer dyes equipped with triphenylamine donor groups, which are currently among the most efficient sensitizers in DSC. Cobalt-based redox couples were shown to be viable alternatives to the standard iodide / triiodide system, provided that a dye with suitable steric properties is used. Detailed in-situ studies of DSCs have revealed the occurrence of a Stark-effect, where an internal electric field changes the absorption of the dye. This effect can be used to provide fundamental understanding of the DSC. In development work, CMD has shown that monolithic DSC is a viable PV technology, although further development and testing is required.

\section{Acknowledgement}

We thank the Swedish Energy Agency, the Swedish Research Council, Vinnova, BASF SE and the Knut and Alice Wallenberg foundation for financial support.

\section{References}

[1] A. Hagfeldt, G. Boschloo, L. Sun, L. Kloo, H. Pettersson, Chem. Rev. 110 (2010) 6595.

[2] G. Smestad, C. Bignozzi, R. Argazzi, Sol. Energy Mater. Sol. Cells 33 (1994) 253.

[3] J. M. Kroon, N. J. Bakker, H. J. P. Smit, P. Liska, K. R. Thampi, P. Wang, S. M. Zakeeruddin, M. Grätzel, A. Hinsch, S. Hore, U. Würfel, R. Sastrawan, J. R. Durrant, E. Palomares, H. Pettersson, T. Gruszecki, J. Walter, K. Skupien, G. E. Tulloch, Prog. Photovolt.: Res. Appl. 15 (2007) 1.

[4] D. P. Hagberg, T. Edvinsson, T. Marinado, G. Boschloo, A. Hagfeldt, L. Sun, Chem. Commun. (2006) 2245. 
[5] G. Boschloo, T. Marinado, K. Nonomura, T. Edvinsson, A. G. Agrios, D. P. Hagberg, L. Sun, M. Quintana, C. S. Karthikeyan, M. Thelakkat, A. Hagfeldt, Thin Solid Films 516 (2008) 7214-7217.

[6] S. M. Feldt, E. A. Gibson, E. Gabrielsson, L. Sun, G. Boschloo, A. Hagfeldt, J. Am. Chem. Soc. 132 (2010) 16714.

[7] G. Boschloo, A. Hagfeldt, Acc. Chem. Res. 42 (2009) 1819.

[8] K. Fredin, E. M. J. Johansson, T. Blom, M. Hedlund, S. Plogmaker, H. Siegbahn, K. Leifer, H. Rensmo, Synth. Metals 159 (2009) 166.

[9] U. B. Cappel, E. A. Gibson, A. Hagfeldt, G. Boschloo, J. Phys. Chem. C 113 (2009) 6275.

[10]U. B. Cappel, M. H. Karlsson, N. G. Pschirer, F. Eickemeyer, J. Schöneboom, P. Erk, G. Boschloo, A. Hagfeldt, J. Phys. Chem. C 113 (2009) 14595.

[11] M. Hahlin, E. M. J. Johansson, S. Plogmaker, M. Odelius, D. P. Hagberg, L. Sun, H. Siegbahn, H. Rensmo, Phys. Chem. Chem. Phys. 121507.

[12] M. Zuleta, S. Yu, S. Ahmadi, G. Boschloo, M. Göthelid, A. Hagfeldt, Langmuir 26 (2010) 13236.

[13]G. Boschloo, L. Häggman, A. Hagfeldt, J. Phys. Chem. B 110 (2006) 13144.

[14]Z. Yu, M. Gorlov, G. Boschloo, L. Kloo, J. Phys. Chem. C ASAP.

[15]U. B. Cappel, S. M. Feldt, J. Schoneboom, A. Hagfeldt, G. Boschloo, J. Am. Chem. Soc. 132 (2010) 9096.

[16]H. Pettersson, T. Gruszecki, R. Bernhard, L. Häggman, M. Gorlov, G. Boschloo, T. Edvinsson, L. Kloo, A. Hagfeldt, Prog. Photovolt: Res. Appl. 15 (2007) 113-121.

[17]H. Pettersson, T. Gruszecki, C. Schnetz, M. Streit, Y. Xu, L. Sun, M. Gorlov, L. Kloo, G. Boschloo, L. Häggman, A. Hagfeldt, Prog. Photovolt.: Res. Appl. 18 (2010) 340. 